\title{
Un nuevo enfoque sobre la esquizofrenia
}

\section{Carmen Varela Garciay Robert P. Liberman}

\author{
Residencia Provincial Santa Maria de las Nieves de Alavay Universidad de California en Los \\ Angeles
}

\section{INTRODUCCION}

La esquizofrenia, término acuñado a principios del siglo $\mathrm{XX}$, es una enfermedad que existe en todas las culturas y en todas las épocas. Aproximadamente el 1 por 100 de la población mundial sufre esquizofrenia, cuyas víctimas proceden indistintamente de las tribus primitivas y de las culturas más tecnificadas, de grupos económicamente débiles y de aquellos que gozan de mayor fortuna. Esta enfermedad mental está considerada como la más grave, dado que afecta a todos los aspectos de la personalidad del individuo (a cómo piensa, siente, actúa, trabaja, juega y cuida de sí mismo). El aspecto básico o central de la esquizofrenia es la huida que el sujeto hace de la realidad a través de sus pensamientos, sentimientos y comportamiento y a través de experiencias que le son muy personales y no comparte con los demás.

Hasta épocas muy recientes se usó una definición de la esquizofrenia totalmente vaga, lo que cristalizó en un diagnóstico de escasa fiabilidad y una sobreestimación de la aparición de los trastornos, pero las múltiples colaboraciones internacionales en los últimos diez años han resultado efectivas a la hora de especificar los criterios diagnósticos de la esquizofrenia. Este mayor rigor y esta clasificación operativa se refleja en el nuevo Diagnostic and Statistical Manual (DSMIII) de la Asociación de Psiquiatría Americana, de reciente publicación. El análisis de los síntomas, elicitados mediante una cuidadosa entrevista del paciente, puede revelar:

- Delirios de estar controlado por fuerzas externas.

- El sentimiento de que los pensamientos estén siendo emitidos a través de centros radiofónicos al mundo externo, por lo que otras personas pueden oirlos.

- La vivencia de que alguien inserte los pensamientos en su propia mente.

- La creencia de que los pensamientos sean extirpados de su cabeza.

- Alucinaciones auditivas, en las que una voz mantiene comentarios constantes sobre su comportamien- 
to o sus pensamientos; o dos o más voces que llevan a cabo estas conversaciones entre ellas.

Existen muy pocas generalizaciones para todos los esquizofrénicos. De hecho, los patrones sintomatológicos y las experiencias irreales varian enormemente de un paciente a otro. La gravedad, duración e incapacidades de la enfermedad no son en absoluto uniformes, e incluso en un mismo individuo los síntomas aparecen y desaparecen, siendo muy pocos los sujetos que sufren los síntomas de la esquizofrenia veinticuatro horas al día o trescientos sesenta y cinco días al año. Esta exacerbación y remisión de los síntomas y los patrones variables e impredictibles del desarrollo sintomatológico vienen a ser la regla. Así, en algunos sujetos, los síntomas se desarrollan lenta y perezosamente, mientras que en otros la ruptura con la realidad es rápida $y$ desgarradora.

Aunque diversos estudios con gemelos y adoptivos han descubierto un componente genético en la esquizofrenia, parece que se trata de una tendencia o susceptabilidad más que de una etiología directa. Las influencias ambientales son también importantes en la precipitación de los sintomas, puesto que solamente un 25 por 100 de todos los pares de gemelos idénticos desarrollan ambos la enfermedad. Por otra parte, se afirma que ciertos atributos en la personalidad de los padres contribuyen a la esquizofrenia, pero la investigación realizada en este sentido no ha podido confirmar este punto, probablemente las influencias familiares jueguen un mayor papel en la evolución o curso de la enfermedad, una vez establecida, que en el desarrollo inicial de los síntomas.

\section{TRATAMIENTO DE LA ESQUIZOFRENIA}

Puesto que la esquizofrenia no es una condición unitaria y se expresa de forma diferente en individuos diferentes, con síntomas que cambian frecuentemente, no es sorprendente que no haya aparecido hasta ahora un tratamiento único mejor que los demás; en cambio, se ha visto que varios tratamientos biológicos y psicosociales pueden ayudar de forma efectiva. Así el conjunto de drogas incluido en los neurolépticos - también conocidos con el nombre de phenothiazinas y tranquilizantes mayores - han mostrado ser valiosas para la mayor parte de esquizofrénicos y forman el tratamiento más estandarizado para dicha enfermedad; tal medicación ha ayudado a reducir el número de esquizofrénicos hospitalizados y a fomentar el desarrollo de programas de rehabilitación orientados a la comunidad, de hecho debilitan o eliminan los síntomas, permitiendo al individuo afligido pensar de forma más clara y aprender más del ambiente.

\section{LIMITACION DE LOS NEUROLEPTICOS EN EL TRATAMIENTO DE LA ESQUIZOFRENIA}

A pesar de las esperanzas y expectativas puestas en ellos, los neurolépticos no han proporcionado un tratamiento completo y satisfactorio para la esquizofrenia. Esta medicación antipsicótica ha reducido la duración de la estancia en el hospital, pero ha aumentado el uso de la "puerta giratoria» en el patrón de altas y reingresos en las dependencias psiquiátricas. En un estudio reciente de las admisiones consecutivas de esquizofrénicos en un centro de salud mental típico, se encontró que el 45 por 100 eran reingresados en los seis meses siguientes al alta. Esto significa que el tratamiento farmacológico alivia enormemente el sufrimiento, pero no previene una recaída posterior y la rehospitalización. Estas recaídas, por otra parte, pueden ser incluso más perturbadoras que una cronicidad sostenida y conducen a una gran desmoralización de los pacientes, sus familias y el personal implicado en el tratamiento; esta desmoralización de por sí tiene una 
influencia adversa en los esfuerzos terapéuticos.

Por otra parte, otros dos factores importantes limitan el uso de neurolépticos y señalan la necesidad de intervenciones psicosociales efectivas, estos factores son los efectos secundarios y la resistencia del paciente a tomar este tipo de drogas. Los efectos secundarios de los neurolépticos, especialmente los espasmos neuromusculares, temblores, inquietud y rigidez, contribuyen a la renuncia del paciente a mantener el régimen medicamentoso. Incluso entre los que toman la medicación de forma fiable, el 48 por 100 recae en menos de dos años. Por tanto, los neurolépticos a lo sumo retrasan, pero no previenen la recaída. Las drogas no enseñan las habilidades necesarias para la vida cotidiana, ni mejoran la calidad de vida del individuo, sino de una forma indirecta a través del desplazamiento de síntomas, por lo que la mayor parte de los pacientes esquizofrénicos necesitan aprender o reaprender habilidades sociales y personales para sobrevivir en la comunidad.

\section{FACTORES FAMILIARES EN LA RECAIDA DE LOS ESQUIZOFRENICOS}

Una serie de estudios realizados en los últimos 20 años, estudios que comenzaron en Londres y hoy se continúan en investigaciones patrocinadas por la OMS y los Institutos Nacionales de Salud Mental en Dinamarca, Colombia, la India y los Estados Unidos, realzan la importancia del clima emocional en la familia en la evolución de la enfermedad esquizofrénica y parece evidente que el proceso interpersonal dentro de la familia es uno de los determinantes más poderosos en la recaída de una persona con una enfermedad esquizofrénica establecida. De hecho, si decidimos usar un predictor único de una recaída sintomatológica, el mejor es el nivel emocional expresado por un familiar al paciente, para ello se utiliza la «emoción expresada», que consiste en registros de los, sentimientos y actitudes de los familiares hacia el paciente y que reflejan la crítica, desilusión, hostilidad, solicitud, preocupación extrema, sobreprotección y un ambiente demasiado emotivo en general.

Los familiares, aparte del paciente, expresan los sentimientos espontáneamente durante una entrevista semiestructurada, con preguntas detalladas sobre las actividades familiares, la frecuencia de disputas, el aislamiento social y la evolución de la enfermedad del paciente. Para medir la emoción expresada por un familiar, el entrevistador evalúa los elementos no-verbales, asi como los contenidos verbales semánticos de las respuestas del familiar. Se sitúa el énfasis en los aspectos vocales del discurso - tono, "pitch", intensidad, rapidez y fluidez - en el registro de diversas escalas que reflejan las cualidades emocionales. El tono de voz o las verbalizaciones de resentimiento claras y no ambiguas, la desaprobación o disgusto sobre algo que el paciente ha dicho o ha hecho contribuye a la identificación de un comentario crítico.

Tres estudios de réplica diferentes han revelado que los pacientes que vuelven a familias que expresan un alto nivel de crítica y están rodeados de un ambiente altamente emocional, recaen cuatro veces más que aquellos que vuelven a familias con bajos índices en estas categorias de expresión emocional. El 51 por 100 de los pacientes que volvieron a hogares donde un miembro familiar poseía una emoción expresada alta, recayeron antes de los nueve meses de salir del hospital; mientras que sólo recayó el 13 por 100 de aquellos que volvieron a hogares de emoción expresada baja. $\mathrm{El}$ diagnóstico inicial de la esquizofrenia y las determinaciones clínicas de la recaida fueron realizados rigurosamente y de forma fiable en base al Present State Examination (PSE).

El uso de medicación antipsicótica y un contacto cara a cara de menos de 35 horas semanales protege a los sujetos esquizofrénicos de la recaida en familias de alta expresión emocional. Los pacientes 
que viven con familiares con un alto indice de expresión emocional, que pasan mucho tiempo con ellos y no están protegidos por una terapia medicamentosa, tienen un pronóstico muy pobre, con un 92 por 100 de recaídas. Esta tasa de recaidas disminuye cuando uno de los factores protectores está operando. El mejor pronóstico - equivalente al de los pacientes que retornan a familias con un índice de emoción expresada bajo- es para aquellos en los que operan ambos factores protectores.

\section{IMPLICACIONES CLINICAS: ENTRENAMIENTO EN HABILIDADES SOCIALES Y TERAPIA FAMILIAR}

Es significativo el hecho de que a través de los estudios sobre emoción en familias de esquizofrénicos, hoy es posible identificar de forma objetiva un grupo de sujetos esquizofrénicos con alto riesgo de recaída. El grupo que presenta un mayor riesgo es el de hombres solteros que viven con sus padres, los cuales manifiestan un alto grado de emoción expresada. ¿Qué tipo de intervención clínica sería apropiada, entonces, para este grupo? Considerando las variables que mediatizan la recaida, se necesitaria mantener la medicación antipsicótica y reducir el contacto cara a cara con sus familiares después del alta. Sin embargo, y a pesar de que algunos procedimientos especiales (como el uso de drogas antipsicóticas de efecto retardado y técnicas de reforzamiento) pueden garantizar el uso continuado de la medicación, los efectos secundarios y la falta de colaboración del enfermo limitan el éxito de esta vía terapéutica. Por otra parte, la reducción del contacto cara a cara es más fácil de decir que de hacer, puesto que muchas de las familias con un alto índice de emoción expresada poseen una «ligazón" entre sus miembros alimentada por años de dependencia y gratificaciones mutuas.

Otros dos enfoques que se están evaluando actualmente en el «Camarillo/U-
CLA Mental Health Clinical Research Center for the Study of Schizophrenia» y la Residencia Provincial Santa María de las Nieves de Alava, incluyen la reducción del grado de emoción expresada por los familiares mediante un tipo especial de terapia familiar conductual por una parte, y el fortalecimiento de habilidades sociales y de comunicación del paciente a través de un programa cuyo objetivo es posibilitar al paciente un mejor entendimiento con sus familiares o motivarle a dejar su familia y ser más autónomo, por otra.

Si admitimos la teoría actualmente popular de la diátesis estressante de la esquizofrenia, es necesario analizar detalladamente el proceso por el que los esstresantes ambientales, como puede ser un alto grado de emoción expresada, que actúan sobre un sujeto vulnerable, producen de hecho una crisis en el funcionamiento personal y social y una exacerbación sintomática o recaída. Aunque es evidente que la medicación neuroléptica reduce la vulnerabilidad del sujeto esquizofrénico a la recaida y protege a la persona de un impacto esstresante de los acontecimientos cotidianos y de un alto grado de emoción expresada, ¿por qué estos sujetos sucumben ante esstresantes que otros pasan por alto o pueden soportarlos? Una posibilidad es que los síntomas esquizofrénicos, cualesquiera que sean sus mecanismos biológicos, se manifiestan cuando el sujeto es abrumado por circunstancias situacionales desafiantes y por problemas para los que no posee los recursos interpersonales suficientes para revolverlos.

Desde este punto de vista, un incremento o reaparición de los síntomas en una persona vulnerable a la esquizofrenia es resultado del equilibrio o interacción entre la cantidad de estressantes cotidianos y la capacidad del sujeto de resolver problemas. En este sentido, tanto un cambio demasiado grande en el estressante - por ejemplo, una fuerte crítica de un familiar al que el esquizofrénico está emocionalmente muy apegadocomo una capacidad muy pobre a la 
hora de resolver problemas - por ejemplo, una persona que siempre responde a las situaciones nuevas con aislamiento social - pueden conducir a una sintomatología florida. Este modelo de formación de sintomas es aplicable de igual manera a otros trastornos médicos y psiquiátricos, por ejemplo, si la diátesis de una persona es para úlceras pépticas o hipertensión, los estressantes excesivos o una habilidad deficiente en solución de problemas pueden conducir a la aparición o reaparición de los síntomas en una persona particularmente susceptible. Nuestra estrategia para reducir las recaidas es fortalecer la competencia en solución de problemas y las habilidades sociales de los esquizofrénicos a través de un entrenamiento directo y mejorar el clima emocional dentro de la familia mediante una terapia que les enseñe habilidades de comunicación. Tales intervenciones fueron ya empleadas durante un periodo de tres meses de hospitalización y evaluadas antes, después del tratamiento y durante un programa de seguimiento de nueve meses.

El entrenamiento en habilidades sociales intenta incrementar la competencia funcional de un paciente en situaciones cotidianas. Este entrenamiento es positivo y educativo y presupone que cada sujeto actúe lo mejor que pueda, dada su dotación biológica, limitaciones cognitivas y experiencias previas en aprendizaje social. Cuando el "mayor esfuerzo" de un sujeto falla en la consecución de sus objetivos y necesidades interpersonales, esto es un indicador de un déficit especifico en el repertorio de dicho sujeto. Este déficit puede provenir de una diátesis biológica para la esquizofrenia, o de una falta de experiencia, o de cantidades extremas de estrassantes sociales. Cualquiera que sea el origen de este déficit, de todos modos, los terapeutas deberían ayudar a la persona a superar o compensar parcialmente tales déficit mediante un entrenamiento sistemático en respuestas alternativas más efectivas, cuyo objetivo seria fortalecer la capacidad del paciente para sobrellevar una gran variedad de si- tuaciones interpersonales. Las secuencias de entrenamiento se basan en aspectos situacionales específicos de intercambios emocionales, sociales e instrumentales entre las personas. Este entrenamiento, por supuesto, puede realizarse en vivo incluyendo a aquellas personas que tienen relación con los problemas cotidianos del paciente o puede realizarse mediante el uso de técnicas de rol-playing.

El entrenamiento en habilidades sociales se centra en la atención, percepción e interpretación ajustada de las señales ambientales, en la generación de alternativas posibles a la hora de actuar, y en la emisión de respuestas sociales con mayor expresividad verbal y no-verbal; también incluye una serie de habilidades conversacionales: escucha activa, captación y clasificación de niveles de auto-apertura apropiados, como cambiar de tema y como terminar una conversación y otra serie de habilidades para sobrevivir en la comunidad: cocinar, limpiar, planificación económica y recreacional, etc.

Estos pacientes, al mismo tiempo, se reúnen semanalmente en grupos de tres familias. Cada grupo tiene tres colíderes que dirigen conjuntamente las sesiones teóricas y luego trabajan separadamente con cada familia en sesiones prácticas durante la segunda mitad de cada reunión de dos horas. Las dos primeras sesiones son sesiones teóricas sobre la esquizofrenia, sus causas, evolución y tratamientos. La información se traduce a un nivel fiable de entendimiento, presentando la esquizofrenia como un trastorno que acarrea problemas graves en la vida cotidiana - trabajo, cuidado personal, socialización, pensamientos y sentimientos- Se ayuda a las familias a desechar sus sentimientos de culpa, de responsabilidad exagerada, confusión y desesperanza. A los pacientes se les anima a que analicen detalladamente sus problemas, a que obtengan una esperanza razonable sobre modestos progresos en su acomodación a las circunstancias dañinas. Una sesión se centra en el uso asertivo de los recursos comunitarios y psiquiátricos durante el periodo poshos- 
pitalario. Otras siete sesiones se basan en habilidades de comunicación familiar, las cuales se describen, racionalizan y demuestran para ensayarlas luego en los grupos familiares - expresión de sentimientos positivos y feed-back, realizar demandas positivas, desarrollar habilidades de escucha, expresión de sentimientos negativos y adaptación a los síntomas-. Con las familias se usa el mismo modelo de solución de problemas que se usa en el entrenamiento de habilidades sociales con los pacientes. Cada paciente y sus familiares trabajan con un terapeuta de la siguiente manera: 1) señalan y especifican los problemas de la vida cotidiana; 2) desarrollan varias opciones o respuestas alternativas para solucionar el problema; 3) sopesan los pros y contras de cada alternativa, especialmente en términos de las consecuencias potenciales en el caso de realizarla; 4) escogen la alternativa que parece más razonable; 5) deciden cómo poner en práctica la respuesta como familia; 6) proporcionan unos a otros soporte muto en la puesta en práctica; y 7 ) reevalúan el problema después de que han usado la alternativa. La terapia familiar se evalúa por registros anteriores y posteriores de la emoción expresada en las entrevistas familiares, un inventario de conflicto familiar y un cuestionario que mide los conocimientos sobre la esquizofrenia y sus tratamientos.

\section{RESUMEN}

El entrenamiento en habilidades sociales y el programa de terapia familiar están siendo evaluados en un proyecto de investigación que incluye un tratamiento comparativo, con terapia de movilización. En este tipo de terapia los pacientes, asignados al azar y con una esquizofrenia cuidadosamente documentada, practican relajación, footing, reducción de stress, actividades manuales y terapia recreativa. Cada tres meses seis pacientes empiezan el programa terapéutico. Los efectos del tratamiento se evalúan a través de una batería múltiple de instrumentos de evaluación. Esta batería incluye medidas de atención, arousal, asertividad y ansiedad social; tests de rol-play en habilidades sociales en diferentes tipos de situaciones sociales; tests conductuales y cognitivos de solución de problemas interpersonales; y registros de psicopatología y funcionamiento general llevados a cabo por un psiquiatra, enfermeras y familiares. Todas estas medidas se obtienen antes y después del período terapéutico hospitalario, y los registros psicopatológicos de enfermeras y psiquiatra se realizan dos veces al mes. La mayor parte de las medidas se administran de nuevo cada tres meses durante un seguimiento de dos años.

Por tanto, hemos puesto la primera piedra para intentar un enfoque de tratamiento psicosocial de la esquizofrenia con base empirica. Bajo la optimista pretensión teórica de que nuestros pacientes esquizofrénicos tienen capacidad para aprender; que las actitudes y sentimientos de sus familiares pueden cambiar en forma positiva; y que el ambiente donde se desenvuelven - dentro y fuera del hospital - puede diseñarse para superar la diátesis, mediatizada biológicamente, hacia la esquizofrenia, podemos continuar haciendo progresos modestos pero importantes para ayudar a nuestros pacientes esquizofrénicos a llevar unas vidas más reforzantes. 